of an inflammatory process involving the ear, nose, or sinuses, followed after a period by meningitic signs and symptoms. Initial lumbar puncture produces a fluid containing inflammatory cells but with a doubtfully low sugar content and in which and from which no organisms can be seen or cultured. In these cases the possibility of the presence of an intracranial abscess should be carefully considered and investigated but not by further lumbar puncture. The conversion of a patient with an eminently treatable cerebral abscess into one with secondary irreversible brain-stem damage from tentorial pressure cone formation is an iatrogenic tragedy that should be avoidable.

I am grateful to Dr. E. R. Bickerstaff, Mr. J. G. Hamilton, Mr. J. M. Small, and Mr. E. A. Turner for allowing me to study those cases admitted under their care.

\section{REFERENCES}

Collier, J. (1904). Brain, 27, 490

Du Boulay, G. H. (1965). Principles of X-ray Diagnosis of the Skull. London.

Finney, L. A., and Earl Walker, A. (1962). Transtentorial Herniation. Springfield, Illinois.

Jefferson, G. (1938). Arch. Neurol. Psychiat. (Chic.), 40, 857. Korein, J., Cravioto, H., and Leicach, M. (1959). Neurology (Minneap.),
9, 290 .

Lilia, B. (1948). Acta radiol. (Stockh.), 30, 129.

Masson. C. B. (1929). Arch. Neurol. Psychiat. (Chic.), 21, 1141.

Meyer, A. (1920). Arch. Neurol. Psychiat. (Chic.), 4, 387.

Nordmark, B. (1949). Acta radiol. (Stockh.), 32, 461.

Norlén, G., and Wickbom, I. (1958). 7. Neurol. Neurosurg. Psychiat., 21, 1 .

Smyth, G. E., and Henderson, W. R. (1938). F. Neurol. Psychiat., 1,

Young, B. R. (1949). Radiology, 53, 625.

\title{
Width of Clavicular Cortex in Osteoporosis
}

\author{
H. C. ANTON,* M.B., ch.B., F.F.R.
}

\begin{abstract}
Summary : Thinning of the upper cortex of the clavicle, $\checkmark$ measured on a standard chest radiograph, may help in the diagnosis of osteoporosis. No precise level at which osteoporosis occurs can be given, but a reading of $1.5 \mathrm{~mm}$. or under is indicative of osteoporosis, while a smaller incidence is associated with readings of $2 \mathrm{~mm}$. and above. There is a significant correlation between thinning of the clavicular cortex and other radiological indications of osteoporosis. Thinning may occasionally point to unsuspected bone disease, in osteomalacia as well as in osteoporosis. As chest radiographs are taken in a high proportion of both outpatients and inpatients, the method has a wide applicability.
\end{abstract}

\section{Introduction}

Many excellent radiological methods for assessment of osteoporosis have been developed, such as the osteoporosis index of Barnett and Nordin (1960) and the ulnar densitometric method (Doyle, 1961). Such methods are rather time-consuming, and are generally used only in individuals or in groups already suspected of osteoporosis, or thought to be liable to it. Measurement of the cortical thickness of the radius, described by Meema (1963), is a valuable though simple method, but again radiographs of the forearm are not likely to be available save by special request. It seemed that there was a place for a simple method which might help in the detection of osteoporosis from a widely employed radiograph as well as in the assessment of its severity. This paper reports the value of estimations of the width of the cortex of the clavicle from routine chest films.

\section{Methods}

The thickness of the upper cortex of either clavicle (henceforward termed the C.C.T.) has been measured, on a standard posteroanterior chest radiograph, to the nearest $0.5 \mathrm{~mm}$. using an ordinary rule. The midpoint of the clavicle is taken for the reading, as the cortex thins appreciably towards the medial aspect of the clavicle and may show a double cortex at its lateral aspect. Furthermore, in the outer half of the cortex there is occasionally a localized cortical thickening associated with a nutrient foramen. The lower cortex proves unsuitable for measurement, as it is often poorly defined and sometimes, though rarely, is absent when other bones show normal cortices. It was decided not to compare the C.C.T. with the total width of the clavicle, as it seemed unlikely that this ratio would show any advantage, and because it would detract from the simplicity of the method.

The investigation is in two main parts. In the first part an attempt was made to find if there were differences in the C.C.T. related to the age and sex of the patient. For this purpose measurements of the C.C.T. were made on 120 randomly selected chest radiographs from both inpatients and outpatients during routine reporting in a general hospital. The readings were made before the age of the patient was noted. As a considerable proportion of the patients were elderly it is inevitable that some cases of osteoporosis will be included in the series. The results are given in Table I.

As the first part of the study suggested that a C.C.T. of $1.5 \mathrm{~mm}$. or under might be associated with osteoporosis, in that none of those below 40 years old showed such a figure, a record of consecutive cases with such a C.C.T. -56 in all, including the 15 relevant cases from the first part of the study -was kept over a period of several months. Their radiographs and case records were studied to determine whether a low C.C.T. was associated with other evidence of osteoporosis. This forms the basis of the second part of the study, the findings being presented in Table II.

Of the 56 cases with a C.C.T. of $1.5 \mathrm{~mm}$. or under, 25 were excluded because no radiographs other than chest ones were available. Five further cases were excluded from Table II, as only spine films were available, and these showed no evidence of wedging or biconcavity of the vertebral bodies. They were, however, included as negative cases in Table III. The work of Doyle, Gutteridge, Joplin, and Russell Fraser (1967) indicates that the subjective assessment of spinal osteoporosis is apt to 
be fallacious, and therefore it seems proper to include only cases with objective evidence of spinal osteoporosis, in the form of structural deformities such as biconcavity or wedging due to compression fractures.

Finally, to compare with the cases in Table II, a similar study of cases with C.C.T.s of $2 \mathrm{~mm}$. and over was carried out concurrently. The findings are utilized in Tables III and IV.

\section{Results}

Table I gives the relation between the C.C.T. and the age and sex of the patient.

TABle I.-Clavicular Cortical Thickness Related to Age and Sex in 120 Randomly Selected Patients

\begin{tabular}{|c|c|c|c|c|c|c|c|c|c|c|c|c|}
\hline \multirow{2}{*}{\multicolumn{2}{|c|}{ Age }} & \multicolumn{9}{|c|}{ No. of Patients with C.C.T. (mm.) } & \multicolumn{2}{|c|}{ C.C.T. (mm.) } \\
\hline & & 1 & 1.5 & 2 & 2.5 & 3 & 3.5 & 4 & 4.5 & Total & Mean & S.D. \\
\hline ale & $\begin{array}{l}20-39 \\
-40-59 \\
60+ \\
20-39 \\
-40-59 \\
60+\end{array}$ & 4 & $\begin{array}{l}7 \\
1 \\
3\end{array}$ & $\begin{array}{r}3 \\
6 \\
11 \\
2 \\
2 \\
5\end{array}$ & $\begin{array}{r}3 \\
8 \\
4 \\
6 \\
3 \\
10\end{array}$ & $\begin{array}{l}6 \\
4 \\
3 \\
9 \\
4 \\
8\end{array}$ & $\begin{array}{l}1 \\
1 \\
2 \\
1\end{array}$ & 1 & 1 & $\begin{array}{l}14 \\
19 \\
29 \\
20 \\
12 \\
26\end{array}$ & $\begin{array}{l}2 \cdot 78 \\
2 \cdot 50 \\
1.91 \\
2 \cdot 87 \\
2 \cdot 70 \\
2 \cdot 44\end{array}$ & $\begin{array}{l}0.56 \\
0.42 \\
0.57 \\
0.54 \\
0.84 \\
0.48\end{array}$ \\
\hline
\end{tabular}

In females the difference between the means of the young adult group and the middle-aged group is not statistically significant (S.E. $=0.17 \mathrm{~mm}$.). Comparison of the elderly group with the middle-aged one shows that the difference between the means is significant at the $99 \%$ level (S.E. $=0.14 \mathrm{~mm}$.)

In males the difference between the means of the young adults and the middle-aged is not significant (S.E. $=0.27 \mathrm{~mm}$.), but the difference between the means of the middle-aged and the elderly is significant at the $95 \%$ level (S.E. $=0.26 \mathrm{~mm}$.).

The differences between the means of the young adults and between the means of the middle-aged in the two sexes are not significant; but the difference between the means of the two elderly groups is significant at the $99 \%$ level (S.E. $=0.14 \mathrm{~mm}$.).

Table II presents, for cases with a C.C.T. of $1.5 \mathrm{~mm}$. and under, the results of radiological assessment of osteoporosis and other relevant findings from radiographs of the spine, hand, and femur, and lists any known aetiological factors found in the case records.

TABLE II.-Findings Associated with a C.C.T. of $1.5 \mathrm{~mm}$. or Less in a

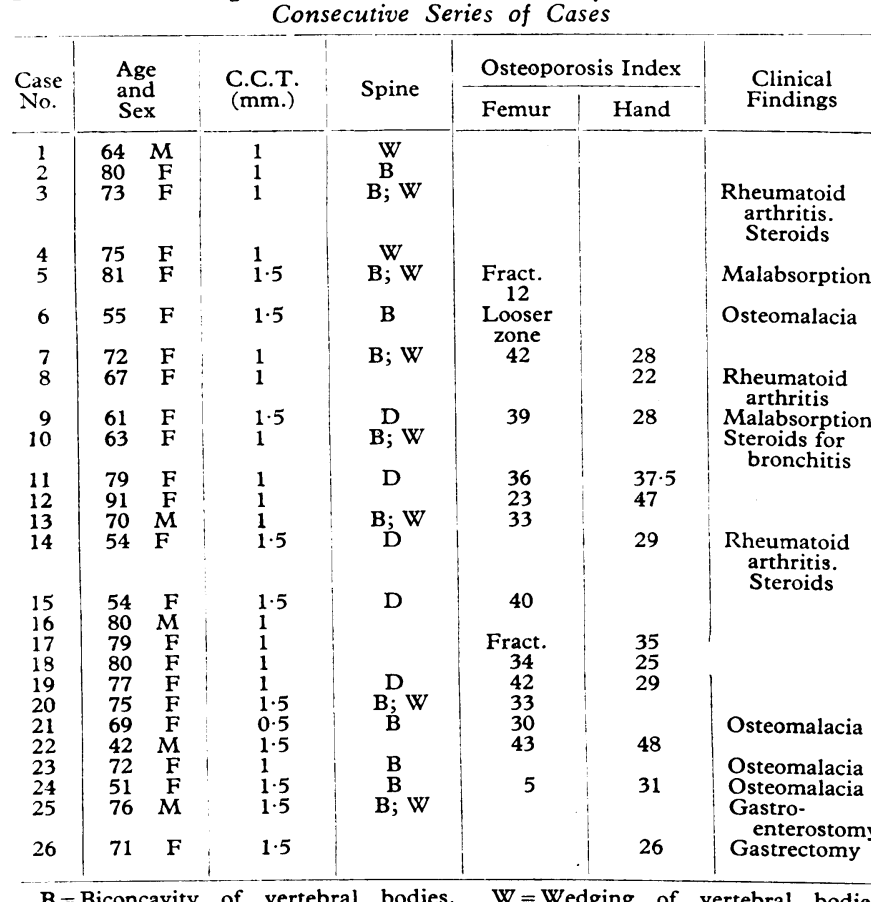
$\mathrm{B}=$ Biconcavity of vertebral bodies. $\mathrm{W}=$ Wedging of vertebral bodies
Cases in which the clinical findings are left blank are assumed to have an idiopathic osteoporosis.

The osteoporosis index is a measure of the ratio of the combined cortical thickness of the total diameter of a bone, measured on an anteroposterior radiograph. The lower limits of normal are 44 for the hand score and 46 for the femoral score (Barnett and Nordin, 1960).

The diagnosis of osteomalacia was made on clinical and biochemical grounds. The serum calcium and phosphate products were below 27 in all cases and the serum alkaline phosphatase was raised above 12 K.A. units. No biopsy or post-mortem findings are available for any of the cases in Table II.

The main finding in Table II is that the osteoporosis index results are nearly all below the lower limits of normal. The low osteoporosis indices in cases of osteomalacia are also of interest. As expected, the majority of the patients are elderly.

The data from Table II are used to compare the incidence of spinal osteoporosis in cases with different C.C.T.s in Table III, and a simple comparison is given in Table IV for the peripheral scores of the osteoporosis index.

\begin{tabular}{c|c|c|c}
\multicolumn{2}{c}{ TABLE III. - Relation of C.C.T. to Spinal Structural Deformities } \\
\hline C.C.T. (mm.) & No. Positive & Total & Incidence \\
\hline 1.5 and under & 14 & 24 & $58 \%$ \\
2 & 8 & 21 & $38 \%$ \\
2.5 & 3 & 11 & $27 \%$ \\
3 & 1 & 11 & $9 \%$
\end{tabular}

The difference between the incidences corresponding to C.C.T.s of $1.5 \mathrm{~mm}$. and under and $2 \mathrm{~mm}$. is not statistically significant, using the S.E. of differences between proportions S.E. $=14 \%$ ). The difference between the percentages corresponding to C.C.T.s of $1.5 \mathrm{~mm}$. and under and $2.5 \mathrm{~mm}$. is significant at the $95 \%$ level $($ S.E. $=14 \%$ ). The difference between the $58 \%$ and $9 \%$ incidences is significant at the $99 \%$ level (S.E. $=13 \%$ ).

TABLE IV.-Relation of C.C.T. to Peripheral Scores of Osteoporosis Index

\begin{tabular}{|c|c|c|c|c|}
\hline C.C.T. (mm.) & No. & $\begin{array}{c}\text { Mean } \\
\text { Peripheral } \\
\text { Score }(\%)\end{array}$ & S.D. $(\%)$ & S.E. $(\%)$ \\
\hline $\begin{array}{c}1.5 \text { and under } \\
2 \\
3\end{array}$ & $\begin{array}{l}25 \\
25 \\
13\end{array}$ & $\begin{array}{l}31 \cdot 9 \\
50 \cdot 5 \\
56 \cdot 0\end{array}$ & $\begin{array}{c}9.64 \\
10.5 \\
8.61\end{array}$ & $\begin{array}{l}2 \cdot 85 \\
3 \cdot 18\end{array}$ \\
\hline
\end{tabular}

The differences between the means are significant at the $95 \%$ level and at the $99 \%$ level in comparing the means at C.C.T.s of $1.5 \mathrm{~mm}$. and under and $2 \mathrm{~mm}$. The mean peripheral scores in Table IV are calculated from the individual femoral or hand scores and not by combining these two scores as suggested by Barnett and Nordin (1960), for the reason that the numbers with a combined score would be insufficient for analysis.

\section{Discussion}

The significance of cortical thinning is that it indicates a generalized osteoporosis, as the reduction in the bone mass of biopsy specimens occurs equally in both cancellous and cortical bone (Urist, Zaccalini, MacDonald, and Skoog, 1962). Thinning of the upper cortex of the clavicle correlates well with thinning of the cortices of the second metacarpal and the femur, as shown in Table IV.

A discrepancy exists in that Barnett and Nordin (1960) found no relation between their femoral score and ageing, while the C.C.T. falls with age in both sexes, though more strikingly in elderly females. No obvious explanation can be put forward. However, they did show such a relation for the metacarpal 
score. Moreover, Meema (1963) showed a gradual thinning of the cortex of the radius with age in both sexes, to a greater extent in females than in males. Such findings have led several authors to postulate that this gradual loss of bone with ageing is the explanation for so-called senile or postmenopausal osteoporosis (Newton-John and Morgan, 1968). Their finding that the fall in bone mass is linear with respect to age is not fully agreed by other studies. Thus Caldwell (1962), in detailed post-mortem studies of slabs of lumbar vertebrae, using both calcium estimations and radiographic densitometry, found that a parabolic regression curve was more appropriate than a straight line, the osteoporosis becoming progressively more severe with increasing age.

The main limitation of cortical thickness as an indication of osteoporosis is that, since it is a continuous variable, it is not possible to give a precise level at which normality ends and abnormality begins. However, since there were no examples in the age group $20-39$ of a C.C.T. of $1.5 \mathrm{~mm}$. or under, such a level is taken as abnormal at any age. The increased proportion of middle-aged and elderly patients with C.C.T.s of $2 \mathrm{~mm}$. and lower suggests that a C.C.T. of $2 \mathrm{~mm}$. may be associated with osteoporosis, those patients' C.C.T.s in early adult life being perhaps 2.5 or $3 \mathrm{~mm}$. By the same argument a C.C.T. of $2.5 \mathrm{~mm}$. in middle age may represent an initial level of $3.5 \mathrm{~mm}$. The occurrence of such a fall may explain the presence of osteoporotic spinal deformities, though in decreasing incidence, associated with C.C.T.s of $2 \mathrm{~mm}$. and over shown in Table III

It might be assumed that the $58 \%$ incidence of spinal biconcavity or wedging due to compression fractures at C.C.T.s of $1.5 \mathrm{~mm}$. or under indicates that in the other $42 \%$ evidence of spinal osteoporosis was lacking and that a peripheral osteoporosis could be independent of a spinal one. Several of the cases without such spinal deformities, categorized as doubtful in Table II, showed prominent end-plates, accentuation of vertical trabeculation, or obvious lack of bone density, appearances accepted by some authors-for example, Saville (1967) as evidence of osteoporosis. However, in view of the findings of Doyle et al. (1967), already mentioned, such criteria have not been accepted in this study. Osteoporosis seems inevitable in many of these cases, in that a severe loss of bone mass must precede the development of fracture deformities and biconcavity. This study, then, does not support the concept of independent spinal and peripheral osteoporosis.

The 25 patients with C.C.T.s of $1.5 \mathrm{~mm}$. and below who had no radiographs other than chest ones cannot be assumed to be free of evidence of osteoporosis, as the latter may have no presenting symptoms. The age of this group ranged from 57 to 91 , and $80 \%$ were females. Thus Gershon-Cohen, Rechtman, Schraer, and Blumberg (1953) found asymptomatic spinal fractures in $20 \%$ of males and $29 \%$ of females in the age group 63-95 among healthy residents of a home for the aged.
Biconcavity of the vertebral bodies, the basis for the spine score in the osteoporosis index, is regarded as unreliable by Caldwell (1962), in that a proportion of vertebrae of normal or even high density may show this sign. In practice, however, the significance of biconcavity is usually obvious from other findings. Thus biconcavity is explained by the presence of osteomalacia in four of the cases in Table II, while in another seven cases it is associated with wedging of one or more of the vertebral bodies, strongly suggesting osteoporosis.

Four cases of osteomalacia are included in Table II because of low C.C.T.s, and indeed Case 21 has the lowest C.C.T. recorded by me, $0.5 \mathrm{~mm}$. This is of interest in that contical thinning is not usually described as a sign of osteomalacia, and is not mentioned in the comprehensive review of Steinbach and Noetzli (1964). Two of these cases with the relevant radiographs had low metacarpal and femoral peripheral scores also. That cortical thinning may be a manifestation of osteomalacia per se is suggested by its presence in cases of rickets in infancy and childhood. Caffey and Silverman (1967) described this sign, and the thin cortex is well shown in several of their figures. It seems unlikely that this phenomenon could be due, in this age group, to a coincidental osteoporosis. The four patients in this series, however, are all women over 50 , so that the possibility of a concomitant osteoporosis cannot be excluded in the absence of biopsy findings.

As obscure pains may be the main presenting symptom in both osteomalacia and osteoporosis, it may occasionally happen that the initial diagnosis may be made radiologically. In such cases a low C.C.T. may stimulate a careful review of the radiographs or may indicate a skeletal survey. In a few cases seen since the beginning of this study the C.C.T. has been a major factor in leading to the correct diagnosis.

I wish to thank my clinical colleagues for access to their records, and the radiographers for taking extra films in several cases.

\section{REFERENCES}

Barnett, E., and Nordin, B. E. C. (1960). Clin. Radiol., 11, 166.

Caffey, J., and Silverman, F. N. (1967). Pediatric X-ray Diagnosis, 5th ed. Chicago.

Caldwell, R. A. (1962). F. clin. Path., 15, 421.

Doyle, F. H. (1961). Brit. F. Radiol., 34, 698.

Doyle, F. H., Gutteridge, D. H., Joplin, G. F., and Fraser, R. (1967). Brit. F. Radiol., 40, 241.

Gershon-Cohen, J., Rechtman, A. M., Schraer, H., and Blumberg, N. (1953). F. Amer. med. Ass., 153, 625.

Meema, H. E. (1963). Amer. F. Roentgenol., 89, 1287.

Newton-John, H. F., and Morgan, D. B. (1968). Lancet, 1, 232.

Saville, P. D. (1967). Arthr. and Rheum., 10, 416.

Steinbach, H. L., and Noetzli, M. (1964). Amer. F. Roentgenol., 91, 955.

Urist, M. R., Zaccalini, P. S., MacDonald, N. S., and Skoog, W. A. (1962). F. Bone ft Surg., 44B, 464. 